\title{
Research on Smart Grid Load Forecasting Platform Based on Cloud Computing
}

\author{
Xian Chen ${ }^{a}$, Bo Chen ${ }^{b}$, Xiaozi Cui ${ }^{c}$, Lin Liu ${ }^{d}$ \\ State Grid Henan Electric Power Company Luoyang Power Supply Company, \\ Luoyang 471000, China \\ a luoyangdidiao@sina.com, ${ }^{\text {b } 1015903240 @ q q . c o m ~}$
}

Keywords: Cloud computing, smart grid, load forecasting

\begin{abstract}
Along with the development of Smart Grid, a large number of intelligent terminal equipments are used in power grid. The environment, the means, and the targets of load forecasting will been greatly changed. The load forecasting systems have outstanding heterogeneous problems. Forecasting models cannot control the diversity and complexity, intermittent of the load. Forecasting process needs to weigh the rationality of the mathematical model and the real-time of the calculation frequently. And servers running are at low utilization. But load forecasting is highly required by Smart Grid Forecasting Load (SGFL). The development of load forecasting will enter a new stage, including mass data management of load forecasting, the emergence of fluctuating loads, and increasingly higher demand about the density and accuracy of load forecasting and so on, which have important impact on improving the level of load forecasting. With the emergence of cloud computing, power cloud generates. Cloud computing will be able to realize the mass data processing, and achieve reasonable resource allocation. Cloud computing has been widely used in medical, education, and electronic commerce, etc., and also successfully used in power system. Therefore, cloud computing will be adopted in SGLF in the near future.
\end{abstract}

\section{Introduction}

Load forecasting is the traditional research questions in the field of power system, the safe operation of the power system plays an important role. It is energy-efficient scheduling, power generation plans, network planning, stability calculation and other basic work. Numerous examples and data indicate that the accuracy of load forecasting for the rational design of the power structure, the development of fuel program, implementation of energy saving measures to accelerate the pace of economic development in the region is essential.

Power system load forecasting power load based on historical data, economic, social, meteorological and other factors, to explore the influence of variation of electricity load historical data for future load, power load and seek the intrinsic link between the relevant factors, and thus the future the power load of scientific predictions. Power load forecast is to predict the branch school, we can learn from the experience and methods of forecasting in other areas.

\section{Problems of Smart Grid Load Forecasting}

Because Smart Grid load forecasting accuracy, the density of the increasingly high demand, load forecasting difficult. Load forecasting tends to be more traditional forecasting techniques, and smart grid load forecast would predict a fine service to the management level. Relevant literature analysis, load forecasting major face the following problems:

First, from the data storage and processing side, with a large number of terminal devices (smart meters, smart appliances and other embedded) into use and "information technology, digitization, automation, interactive" promote the process of data collection, processing, storage, computing will encounter a problem, we need a new approach. Traditional load forecasting, data processing speed, response time is long, and the various types of information data platform data migration, disaster recovery services cannot meet the current demand. In addition, traditional clustering method will 
load into local optimal solution, even without convergence, the basic load analysis does not proceed; it will seriously affect the load forecasting carried out. Therefore, the data storage and processing is an important part of the smart grid load forecast.

Second, from the mathematical prediction models, along with the smart grid load forecasting accuracy, the density requirements are high, need to study with real-time, adaptive mathematical model. But the factors considered traditional mathematical model is not comprehensive or specific identification of factors is not high, resulting in some extreme points or ability to predict weak at the knee, so that more general factors affecting the load model under not strong. Load analysis model is reflected in the early, mid-prediction algorithm model, late model Load Prediction fine enough.

Third, from the special forecasting objects, random, intermittent loads into load forecasting accuracy at this stage leads to instability. This often means that partial load in the typical large user load, high speed electrified railway load, distributed load wind farm power generation, solar photovoltaic power generation load, electric vehicle charging load and the like. Predict the impact load above the level of the prediction accuracy of the region with the development of smart grid load gradually increased, these loads tend to use the Internet or at the substation load dedicated network, predicted results will directly affect the flow calculation, security check and so on.

Fourth, from the load forecasting system is concerned, the majority of the existing load forecasting system is mostly based layer system Browser / Server (B / S) structure, using .NET or J2EE technology. Prediction for different objects (system, buses, large users, etc.), the system is easy to form heterogeneous phenomenon prominent adverse situation unreasonable resource allocation. For example, many provincial power companies tend to adopt a set of system load forecasting, using a system bus load forecasting, or large power customers in the region to adopt a system. And because different build system to system time, system functions, configuration and other difficult to reach consensus. For data sharing, it is basically only at the level of shared predictions.

Fifth, from server health, the load forecasting network, systems, database server CPU utilization is low, most of the time in the idle state or not saturated, a serious waste of resources. Author in the actual system development, operation and maintenance found the general provincial end server deployment system load forecasting using a distributed model in which all parts of the province and end a separate server-side deployment; and bus load forecasting often using a centralized deployment mode, that is, save end and each local shared server side. In fact, all types of server data processing, storage, load analysis, forecasting model calculation, correction calculation does not need to run for a long time, CPU utilization, generally $10 \%$ or less. Overall, server utilization is relatively low load forecasting.

\section{Smart Grid Load Forecast}

Smart Grid has the "information technology, digitization, automation, interactive" of the "four modernizations" characteristics. State Grid Corporation of smart grid definition involves the generation, transmission, substation, distribution, electricity and scheduling six links. The "scheduling" link and smart scheduling proposed new requirements. The power load forecasting is the basic link power dispatch. Therefore, the smart grid load forecasting is part of intelligent scheduling. Smart grid strengthening transition generation dispatching scheduled to load, a load that is not only as a basis for forecasting the development and implementation of energy-saving power generation scheduling plan, but also with the way the load dispatch distributed power output forecast. Therefore, the smart grid load forecasting is an important part of intelligent scheduling.

For load forecast, the current analysis of many forecasting system have different characteristics. With the power industry's rapid development of information technology, smart grid "four modernizations" in the "information" required to overcome the shortcomings of traditional load forecasting, to establish a unified information platform load forecasting. Load analysis, forecasting is based on the data integrity and reliability of the foundation. "Digital" in SCADA, PMU / WAMS is of the digital network to provide data support for load forecasting. In addition, the establishment 
and the "digital public service platform," the use of "digital substation," also can effectively improve the level of load forecasting. "Automation" feature requires extensive use of intelligent devices, improve the accuracy of data collection, also called load forecasting predictive intelligence process, reducing manual intervention, and enhance the adaptability of the model. "Interactive" is not only the requirements of load forecasting to strengthen the prediction target factors interact with each contact, also requires users to determine their own TOU information distributed power output and so on. In particular load forecasting process to consider the needs of the typical user's interactive response (Demand Response, DR) and other factors.

To meet the main characteristics of load forecasting smart grid, and smart grid foundation of facilities and a variety of information services platform to provide effective protection for the load forecast. Load forecasting is an important part of the smart grid and basic content, the smart grid is a data mining system load forecasting environmental and technical support. Therefore, the smart grid and load forecasting are inextricably linked, it is an organic whole. To sum up, smart grid load forecasting is important

\section{Intelligent Load Forecasting Cloud Platform}

Traditional load forecasting work is mainly focused in the development, prediction, and forecast management center to establish a platform technology, to promote the development of load forecasting. However, under the internal system of smart grid electricity demand forecasting is not a single system platform heterogeneity problem outstanding (servers and other infrastructure, diversification, development platforms diversify, diversify data standards, forecasting technological diversification, diversification objects). If you build a cloud platform based on load forecasting data center, we will overcome the data cannot be shared, processing speed and other issues, to cater to the development of smart grid. More and more companies, such as IBM, Adobe, Baidu, Alibaba, etc., using Hadoop deploy cloud platform to address the construction of enterprise information appears in the "data silos, infrastructure dispersed" and other issues.

Smart grid load forecast is for cloud load analysis, forecasting, management and coordination of the results of a private cloud. Currently Google and Amazon cloud-based business is widely used in electronic commerce, communications and other fields, and cannot be directly used for power load forecasting cloud computing platform. Hadoop platform is open source; its unique advantages can a cloud computing services for the smart grid.

Under the smart grid environment, smart devices will be collected in various industries (industry, agriculture, light industry, service industry, residential, commercial, etc.) of data, taking into account different factors in different industries, various factors will be quantified, reflect the model input, load forecasting for various industries. Meanwhile, the forecast results for load management, ordered electricity sector reference.

"Terminal" refers to the user end, different levels of professional control centers (the regulatory profession, Professional, communications professional, automation, multi-disciplinary and professional protection) of user terminal equipment ("four-screen" - computers, televisions, mobile phones, tablet-based), via the Internet (using the current new Internet technology, allows users to remote cloud services via the Internet and practical as possible, between the user and the cloud put wide bridge), sent to the load forecasting cloud request and obtain service.

\section{Summary}

With the gradual development of the smart grid, inadequate load forecasting increasingly significant, traditional forecasting techniques and management practices have been unable to meet current needs. How to improve the stability and load forecasting management load forecasting accuracy is the most important issue under the Smart Grid environmental load forecasting. Cloud computing in the initial use of the power system as well as cloud computing can solve the current serious problems of power system proved cloud computing, smart grid, load forecasting three can closely. Therefore, the study is of practical significance of smart grid load forecasting based on 
cloud computing.

\section{Reference}

[1]Caron E, Desprez F, Muresan A. Forecasting for Grid and Cloud Computing On-Demand Resources Based on Pattern Matching[C] Proceedings of the 2010 IEEE Second International Conference on Cloud Computing Technology and Science. IEEE Computer Society, 2010:456-463.

[2]Zhang H T, Xu F Y, Zhou L, et al. Artificial Neural Network For Load Forecasting In Smart Grid[J]. Machine Learning \& Cybernetics International Conference on, 2010, 6:3200 - 3205.

[3]Yang X, Yuan J, Zhang T, et al. Application of Uncertainty Reasoning Based on Cloud Theory in Spatial Load Forecasting[C]// Intelligent Control and Automation, 2006. WCICA 2006. The Sixth World Congress on. IEEE, 2006:7567 - 7571.

[4]Wang X. Application Research on Electricity Demand Forecasting Based on Gaussian Quadrature Formula[J]. Procedia Engineering, 2011:5574-5578.

[5]Niu D X, Wang Q, Li J. Short Term Load Forecasting Model Based on Support Vector Machine.[J]. Lecture Notes in Computer Science, 2006:880-888.

[6]Li W, Sun W, Liu Z. A novel method of short-term load forecasting based on multiwavelet transform and multiple neural networks[J]. Neural Computing \& Applications, 2013, 22(2):271-277. 